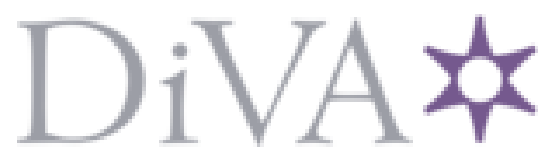

http://www.diva-portal.org

\title{
Postprint
}

This is the accepted version of a paper presented at IEEE Workshop on Computing, Networking and Communications.

Citation for the original published paper:

Hu, Y., Gross, J., Ding, Z. (2013)

The Outage Performance of Realtime Transmission in Multiple Asynchronous Relays Enhanced OFDM System.

In:

http://dx.doi.org/10.1109/ICCNC.2013.6504096

N.B. When citing this work, cite the original published paper.

Permanent link to this version:

http://urn.kb.se/resolve?urn=urn:nbn:se:kth:diva-136859 


\section{The Outage Performance of Realtime Transmission in Multiple Asynchronous Relays Enhanced OFDM System}

\author{
Yulin $\mathrm{Hu}$, James Gross \\ UMIC Research Centre \\ RWTH Aachen University, Germany \\ Email: \{hu|gross\}@umic.rwth-aachen.de
}

\author{
Zhizhong Ding \\ Department of Communication Engineering \\ Hefei University of Technology, China \\ Email: zzding@mail.ustc.edu.cn
}

\begin{abstract}
This paper $^{1}$ considers the outage performance of multiple relays transmission in Orthogonal Frequency Division Multiplexing (OFDM) systems. Relaying has been suggested in general to improve the reliability of wireless systems significantly and is therefore a candidate for wireless industrial communication systems that need to meet tough latency and outage requirements. Different from previous works, this paper takes synchronization mismatch of hardware processing time at multiple relays into account and characterizes the performance impact of such asynchronous relays on the reliability of the system. We focus especially on the case where the transceivers do not have instantaneous channel state information but still need to fullfill tough reliability and latency constraints. We find that with respect to this scenario the synchronization mismatch can lead to a significant loss of reliability even if the individual mismatch of relays is relatively small compared to the cyclic prefix of the OFDM symbol. Consequently, this paper devises countermeasures to efficiently combat the synchronization issues either by introducing a longer cyclic prefix or by decreasing/adjusting the number of active relays. We find that all proposed countermeasures have their application domains which are determined by the average SNR and the severity of synchronization errors.
\end{abstract}

Index Terms-decode-and-forward, multiple relays, average CSI, asynchronous, OFDM, cyclic prefix

\section{INTRODUCTION}

Deploying relays is an effective way to improve the performance [1]-[3] of wireless communication systems. This is has been shown regarding several different performance metrics such as capacity, outage probability (OP) and bit error ratio (BER) [4]-[6]. With respect to real-time transmissions, for example for multimedia or industrial applications, among the mentioned metrics the OP is a very important metric as for such applications data has to be transmitted within a given deadline. While for multimedia applications such a deadline might be somewhat loose, industrial applications typically require stringent deadlines to be met while requiring a high reliability. Naturally, deploying a relay system is therefore a candidate technology to reach the required quality-of-service targets.

\footnotetext{
${ }^{1}$ This work was supported by China Scholarship Council and the DFG Cluster of Excellence on Ultra High-Speed Mobile Information and Communication (UMIC), German Research Foundation grant DFG EXC 89.
}

From previous work it is well known that multiple relays operating cooperatively (in the following referred to as cooperative relays) improve the reliability, i.e. the $\mathrm{OP}$, as the number of relays increases [6]. This is due to two different effects. Most importantly, cooperative relaying increases the spatial diversity of the system as the number of participating relays increases. This simply translates into a significant reliability improvement. Furthermore, cooperative relaying increases the received power of the signal of interest at the receiver which leads to an additional reliability improvement. This increase in reliability is accompanied by an increasing inter-symbol interference (ISI) in general. As more and more relays participate in cooperative relaying, the path diversity from the transmitters to the receiver increases which leads to a larger delay spread experienced at the receiver. However, as long as the relays are not separated too far away from each other, this impact is relatively mild. Nevertheless, this holds only if the relays operate in a fully synchronous manner, i.e. all relays transmit the received packet immediately upon reception.

Unfortunately, in a real system cooperative relays can not achieve complete synchronization. This is mainly due to hardware/software processing issues at the relays. While a slightly unsynchronized system still allows to exploit spatial diversity as well as providing an increased received power at the destination, the synchronization mismatch leads to an additional source of ISI. Hence, the system performance impact of such unsynchronized relays on the reliability of the system becomes important to quantify. This has been recognized by related work already to some extent. For example, [7] studies the synchronization mismatch in multiple amplifyand-forward (AF) relay system. The work assumes that the transmitters have perfect channel state information (CSI) while the synchronization mismatch between transceivers is within a few symbol lengths (i.e. quite significant). In this setting, the authors study joint power loading to enhance the receiver SNR in order to overcome the impact from synchronization mismatch. In a different work, the aurthors of [8] focus on the end-to-end BER performance of a 2 decode-and-forward (DF) relay system. They assume the synchronization mismatch to be within half of a symbol's length. The authors show by means of simulations that the end-to-end BER performance in such 
a system can increase by several orders of magnitude even if the transceivers have perfect CSI available. To mitigate this impact, the authors propose and investigate the application of cooperative space-time coding. Finally, under the assumption of having exactly $4 \mathrm{DF}$ relays, [9] investigates to fully cancel the ISI caused by the mismatch of synchronization. It assumes that the system has perfect CSI while the synchronization mismatch is assumed to be below 15 OFDM samples. The authors propose to fully cancel the ISI by scheduling relays to forward information pairwise and assuming the destination can distinguish different relays's signals by multi-antennas at destination and perfect beamforming technique at relays.

However, when it comes to real time constrained transmissions with very short deadlines, typically the transmitter and/or relays can not be assumed to have perfect CSI. Furthermore, if the reliability requirements are high, potentially many relays are required to ensure the quality of service. With respect to related work it is open what the 'cost of reliability' is as more and more relays are added to the cooperative transmission while synchronization can not be maintained and no up-todate CSI is available at the transceivers.

In this paper, we address these issues in the context of industrial applications which require high reliability as well as very short latencies. Specifically, we focus on the multiDF-relay enhanced two-hop transmission while considering the combined impact from multi-path caused ISI and asynchronous relay nodes. Different from the previous work, the source has no instantaneous CSI which turns the number of active relays in the second transmission phase into a random number. We first analyze the impact from synchronization mismatch on the system performance, and then study two potential countermeasures to be applied. For one countermeasure, we can simply reduce the number of relays to 1 . For the other measure, there is the opportunity to extend the cyclic prefix $(\mathrm{CP})$. We find that both of the two methods can achieve a certain trade-off with respect to the system performance (i.e. reliability), but the corresponding costs of the methods are different: While the former sacrifices the spatial diversity gain from employing multiple relays, the latter shortens the effective transmission time. The contribution of our work is to investigate which strategy achieves a better performance in the considered asynchronous relay system. In particular, notice that we focus on systems that do not have instantaneous CSI at hand while still tough reliability and latency requirements are of interest. This distinguishes our work from the state-ofthe-art.

The rest of the paper is organized as follows. Section II introduces the asynchronous multi-relay system model. And section III, clarifies the problem formulation. Section IV analyzes the outage performance of asynchronous multi-relay system with only average CSI. In Section V we show the performance impact of unsynchronized relays on the reliability of the system, and analyze the performance of different countermeasures to improve the reliability. Finally, we conclude the paper in Section VI.

\section{SySTEM MODEL}

We consider a simple wireless transmission scenario where data packets are to be transmitted from a source $s$ over a set of relays to a distinct destination $d$. We denote the relay nodes by index $i$. The system operates in a slotted fashion. First the data packet is forwarded to the relays during a broadcasting phase of duration $T_{1}$. Next, all relays that have decoded the initial transmission successfully forward the packet to the destination simultaneously during the relaying phase of duration $T_{2}$. The data transmission is constrained by a deadline $T_{\mathrm{d}}$, and we assume that $T_{1}=T_{2}=T_{\mathrm{d}} / 2$. The source node applies a transmit power of $P_{\mathrm{S}}$ while all relay nodes apply a transmit power of $P_{\mathrm{R}}$. Furthermore, we index by $\downarrow i$ the channel from the source to some relay $i$ and by $i \uparrow$ the forwarding channel from some relay $i$ to the destination.

The system applies OFDM as basic transmission scheme where the transmission resources are given by a time/frequency grid of symbols. The symbol duration $T_{\mathrm{s}}$ is fixed for both phases. However, in order to prevent frequency-selective fading, a $\mathrm{CP}$ is inserted. The duration of the prefixes is denoted in the following by $T_{\mathrm{cp} 1}$ and $T_{\mathrm{cp} 2}$. Let us denote by $T_{\mathrm{M}}$ the maximum delay spread (MDS) of the propagation environment. The $\mathrm{CP}$ is set to equal the MDS, i.e. $T_{\mathrm{cp} 1}=T_{\mathrm{cp} 2}=T_{\mathrm{M}}$. So the OFDM symbol length of the two phases is obtained by $T_{\mathrm{s}}+T_{\mathrm{cp} 1}$ and $T_{\mathrm{s}}+T_{\mathrm{cp} 2}$. Furthermore, we denote by $A_{1}$ and $A_{2}$ the total number of subcarriers used during the two phases.

Data transmitted on any channel is received successfully depending on the corresponding signal-to-noise ratio (SNR). The SNR fluctuates randomly and is in general not known by the transmitter or the relays prior to conveying a packet. However, all receivers can perfectly estimate the channel conditions. During the broadcasting phase, the received signal of relay $i$ at time $t$ is given by:

$$
\begin{aligned}
& r_{i}(t)=x(t) \otimes h_{\downarrow i}(t)+n(t) \\
& =\sum_{k} x(t) \otimes \widehat{h}_{\downarrow i, k}\left(t-\delta_{\downarrow i, k}\right)+n(t) .
\end{aligned}
$$

where $\otimes$ denotes the convolution, $x(t)$ denotes the currently transmitted symbol, $h_{\downarrow}(t)$ denotes the channel transfer function and $n(t)$ denotes the complex additive white Gaussian noise process (with mean 0 and variance $\sigma^{2}$ ). The channel transfer function is influenced by path loss, shadowing and fading where the last two are random effects. Due to multi-path propagation the transfer function can be further decomposed into the sum of channel transfer functions $\widehat{h}_{\downarrow i, k}(t)$ over the paths $k$ where $\delta_{\downarrow i, k}$ denotes the delay of the the $k$-th path from source to relay $i$. Therefore, the corresponding SNR at time $t$ and relay node $i$ is given by:

$$
\gamma_{\downarrow i}(t)=\frac{P_{\mathrm{S}} h_{\downarrow i}^{2}(t)}{\sigma^{2}} .
$$

For the shadowing we assume a log-normal distribution while for the fading we assume a Rayleigh-distributed blockfading process which is statistically independent for different 
channels. During a broadcasting or relaying phase the channel states are assumed to be static. Besides, resource blocks in different subcarriers or/and symbols in the same phase are assumed to have the same fading gain in every transmission. From the SNR $\gamma_{\downarrow i}(t)$ we obtain the (random) transmission capacity from the source to relay $i$ by the well known Shannon capacity formula. Taking the duration of the CP into account, the source can transmit $C_{i}$ bits to relay $i$ during the broadcasting phase:

$$
C_{i}=A_{1} \cdot \frac{T_{1}}{T_{\mathrm{s}}+T_{\mathrm{cp} 1}} \cdot \log \left(1+\gamma_{\downarrow i}(t)\right) .
$$

In the system, available relay nodes will try to decode the source's signal and then forward it to the destination. Due to the random channel fluctuations, not all relay nodes from the available relay nodes set (ANS) will be able to decode the signal. We refer to the group of relays that decode the packet successfully as the forwarding relay node set (FNS). Forwarding the packet from the relays in FNS to the destination is not perfectly synchronized in time. We assume this mismatch to stem mainly from slightly different processing durations at the relay nodes, i.e. handling of interrupts when a packet reception of the wireless interface is completed etc. This leads to small time gaps among the overall packet residence times at different relay nodes which leads then to slight mismatches in time when forwarding the data to the destination. In this paper, the mismatch of multiple relays is represented by $\tau_{i, t}$, which is the time difference between the fastest relaying node and relay node $i$ stemming from the hardware processing.

Hence, with the mismatches of hardware processing time (HPT), the joint signal received by the destination is given by: :

$$
y_{\mathrm{d}}(t)=\sum_{i \in \mathrm{FNS}} x(t) \otimes h_{i \uparrow}\left(t-\tau_{i, t}\right)+n(t) .
$$

In the formula above, $h_{i \uparrow}(t)$ denotes the random channel transfer function of the link from relay $i$ to the destination, which is composed of the individual path channel gains $\widehat{h}_{i \uparrow, k}(t)$ with corresponding propagation delay $\delta_{i \uparrow, k}$. So, as with the received signals during the broadcasting phase in Equation (1) the $y_{d}(t)$ can also be expressed as:

$$
y_{\mathrm{d}}(t)=\sum_{i \in \mathrm{FNS}} \sum_{k} x(t) \otimes \widehat{h}_{i \uparrow, k}\left(t-\tau_{i, t}-\delta_{i \uparrow, k}\right)+n(t) .
$$

We further denote by $\gamma_{\mathrm{FNS}}(t)$ the signal to interference plus noise ratio (SINR) of the joint transmission at the destination at time point $t$. Hence, given the definition of the SINR, the joint Shannon capacity in the links from the FNS to the destination $d$ is given by:

$$
C_{\mathrm{d}}=A_{2} \cdot \frac{T_{2}}{T_{\mathrm{s}}+T_{\mathrm{cp} 2}} \cdot \log \left(1+\gamma_{\mathrm{FNS}}(t)\right) .
$$

\section{Problem Formulation}

Conventional literature suggests that the reliability increases as the number of relays increases [6]. However, this does not take the impact of synchronization mismatch into account. While some related work as considered the impact of unsynchronized relays [7]-[9], this always took perfect CSI into account. However, when it comes to industrial applications over wireless networks, typically very tight deadlines have to be met (within a millisecond, for example) while the reliability requirements are also stringent. Hence, for such a setting cooperative relaying is an interesting approach, however the impact due to synchronization mismatch has to be investigated under the assumption that the transmitter/relays do not have instantaneous CSI at hand.

Based on the system model above, this paper mainly focuses on the performance behavior of the source/destination information flow as more and more relay stations are added to the system. Our underlying motivation in this paper is to investigate the following trade-off: The more relays we add to the transmission scenario, the higher the received SNR at the destination will be during the forwarding phase. Also, we add spatial diversity to the broadcasting phase. However, more relays potentially lead to a bigger mismatch in time synchronization between the relays during the forwarding phase. We are hence interested in:

- Characterizing the performance impact of slightly unsynchronized relays on the reliability of the system.

- Devising methods to efficiently combat the synchronization issues either by introducing a longer $\mathrm{CP}$ or by decreasing/adjusting the number of relays to use in the active relay node set (ANS).

In the following we first introduce an OP model for the entire packet transmission from source to destination. Afterwards, we study the above mentioned performance issues by means of simulations.

\section{Outage Probability of Asynchronous Multiple RELAY SYSTEM}

As mentioned in Section II, multiple relay forwarding extends the ISI beyond the duration of $T_{\mathrm{M}}$. This increase of the ISI due to the hardware processing delays leads now to signal components (i.e. paths) that arrive within the CP $T_{\mathrm{M}}$ and other components (paths) that arrive later. In the chosen model, all components that arrive within the CP contribute to the signal of interest while all components that arrive later contribute to interference [10]. Hence, the received signal at the destination is split into two parts: effective signal $y_{\mathrm{e}}(t)$ with power $P_{\mathrm{e}}$ and ISI signal $y_{\mathrm{ISI}}(t)$ with power $P_{\mathrm{ISI}}$.

$$
y_{d}(t)=\sum_{i \in \mathrm{FNS}} y_{d, i}(t)=y_{\mathrm{e}}(t)+y_{\mathrm{ISI}}(t)
$$

$$
\begin{aligned}
& y_{\mathrm{e}}(t)=\sum_{i \in \mathrm{FNS}} y_{\mathrm{e}, i}(t)= \\
& \sum_{i \in \mathrm{FNS}} \sum_{k}^{\delta_{i \uparrow, k}+\tau_{i, t} \leq T_{\mathrm{cp} 2}} x(t) \otimes \widehat{h}_{i \uparrow, k}\left(t-\tau_{i, t}-\delta_{i \uparrow, k}\right)+n(t)
\end{aligned}
$$


TABLE I

$$
\begin{aligned}
& y_{\mathrm{ISI}}(t)=\sum_{i \in \mathrm{FNS}} y_{\mathrm{ISI}, i}(t)= \\
& \sum_{i \in \mathrm{FNS}} \sum_{k}^{\delta_{i \uparrow, k}+\tau_{i, t}>T_{\mathrm{cp} 2}} x(t) \otimes \widehat{h}_{i \uparrow, k}\left(t-\tau_{i, t}-\delta_{i \uparrow, k}\right)+n(t)
\end{aligned}
$$

$y_{d, i}(t)$ is the received signal from relay $i$, while $y_{\mathrm{e}, i}(t)$ is the effective signal part from relay $i$ (the sum of all path components from relay $i$ that have a delay below the CP) and $y_{\mathrm{ISI}, i}(t)$ is the interfering signal part from relay $i$ (the sum of all path components from relay $i$ that have a delay larger than the $\mathrm{CP}$ ). The corresponding power values of the two different components are denoted by $P_{\mathrm{e}, i}$ and $P_{\mathrm{ISI}, i}$. Hence, the SINR of the received signal at the destination is now obtained as:

$$
\gamma_{\mathrm{FNS}}(t)=\frac{\sum_{i \in \mathrm{FNS}} P_{\mathrm{e}, i}}{\sum_{i \in \mathrm{FNS}} P_{\mathrm{ISI}, i}+\sigma^{2}} .
$$

In our two-hop relay system, the source has a packet with size $S$ that needs to be transmitted to the destination. The source can select some relays to be part of the ANS to forward the packet. The OP that relay $i$ can not successfully decode the packet after the broadcasting phase is obtained from Equation (3) by:

$$
\mathrm{P}_{i}=\operatorname{Pr}\left\{C_{i}<S\right\}=\operatorname{Pr}\left\{\gamma_{\downarrow i}<2^{\frac{S \cdot\left(T_{\mathrm{s}}+T_{\mathrm{cp} 1}\right)}{A_{1} \cdot T_{1}}}-1\right\} .
$$

Let us now assume that the source selects $M$ relays into the ANS. When initially transmitting the data to the $M$ relays, not all relays might receive the data successfully. Recall that we refer to the set of remaining relay nodes as FNS. There are $K_{M}=\sum_{i=0}^{M}\left(\begin{array}{c}M \\ i\end{array}\right)$ different combinations for the FNS when the ANS has size $M$. We furthermore denote by $\mathrm{FNS}_{l}$ with $0 \leq l \leq K$ the corresponding set of relay stations that received the packet during the broadcasting phase successfully. Then, the resulting OP when initially selecting $M$ relay stations into the ANS is given by:

$$
\mathrm{P}_{\text {out }}=\sum_{l=0}^{K_{M}}\left(\prod_{\forall i \notin \mathrm{FNS}_{l}} \mathrm{P}_{i}\right)\left(\prod_{\forall i \in \mathrm{FNS}_{l}}\left(1-\mathrm{P}_{i}\right)\right) \mathrm{P}_{l}
$$

where the $\mathrm{P}_{l}$ is the OP of the second slot with the forwarding relay node set $\mathrm{FNS}_{l}$.

$$
\mathrm{P}_{l}=\operatorname{Pr}\left\{C_{d}<S \mid \mathrm{FNS}_{l}\right\}=\operatorname{Pr}\left\{\gamma_{l}<2^{\frac{S\left(T_{\mathrm{s}}+T_{\mathrm{cp} 2}\right)}{A_{2} \cdot T_{2}}}-1\right\}
$$

\section{Performance Evaluation}

The outage performance of the relaying system with synchronization errors derived in the previous section depends on the amount of paths that arrive with a smaller delay than the $\mathrm{CP}$. As this is in general dependent on the random path delays as well as the random hardware processing shift, we evaluate
SIMULATION PARAMETERS

\begin{tabular}{|c|c|}
\hline Antenna & Single and omnidirectional \\
\hline$D_{\mathrm{s} 1}$ & $50 \mathrm{~m}$ \\
\hline$D_{1 \mathrm{~d}}$ & $50 \mathrm{~m}$ \\
\hline$d_{0}$ & $15 \mathrm{~m}$ \\
\hline Source height & $30 \mathrm{~m}$ \\
\hline Relay height & $1.5 \mathrm{~m}$ \\
\hline Destination height & $1.5 \mathrm{~m}$ \\
\hline Power of source & $25 \mathrm{dBm}$ \\
\hline Power of RN & $20 \mathrm{dBm}$ \\
\hline Length of symbol & $16 \mu \mathrm{s}$ \\
\hline NO. of subcarriers per symbol & 512 \\
\hline HPT shift $\tau_{i, t}$ & Uniformly distributed in $[0,2 \mathrm{k}] \mu \mathrm{s}, \mathrm{k}=1,2,4$. \\
\hline Center frequency & $2 \mathrm{GHz}$ \\
\hline Multiple path model & Exponential power delay profile with 10 paths \\
\hline MDS of single link & $4 \mu \mathrm{s}$ \\
\hline &
\end{tabular}

the impact in the following by simulations. First we give an overview of the evaluation methodology, then we discuss the impact of the hardware processing delay and finally discuss possible countermeasures.

\section{A. Evaluation Methodology}

In the simulation, we consider a simple system with $2 n+1$ relays deployed in a vertical straight line with distance $d_{0}$ between every two adjacent relays. As shown in Figure 1, the source, relay 1 and destination are located on the horizontal axis of the system's geometric structure.

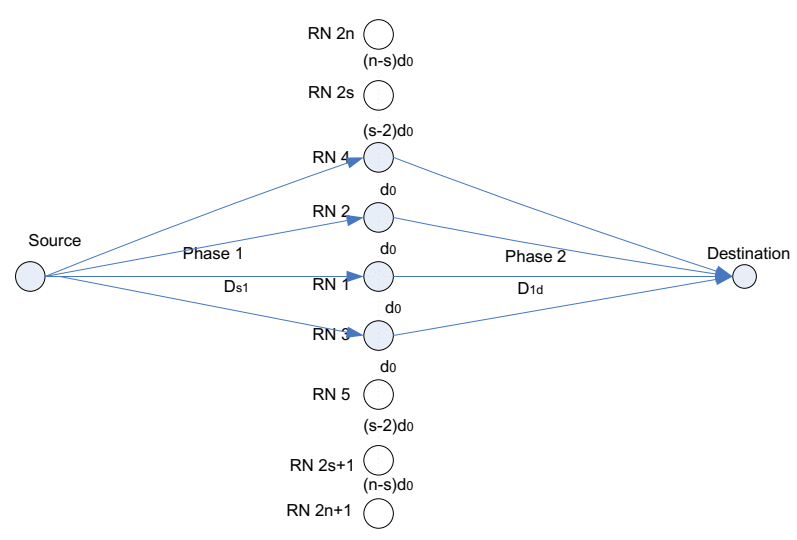

Fig. 1. Multiple relay system

The general simulation parameters are chosen in accordance with the work in [11], [12] and are shown in Table I.

In general, we sample the corresponding distributions of the HPT and of the multipath delay for each presented simulation point 10000 times and construct from the samples the SINR of the links from which we furthermore determine the link capacities. Based on these values we obtain our performance metrics (averaging over the link capacities). We consider two different metrics. The primary performance metric is the OP as obtain by Equations (12) and (13). In addition, we also consider the average capacity (AC) as second performance metric. As the capacity of the broadcasting phase is subjected by the packet size $S$, we choose the AC of the relaying phase as the second performance metric which can simply 
be determined from the 10000 samples of the relaying phase SINR based on Equation (6).

\section{B. Impact of Hardware Processing Time}

In the following we study the $\mathrm{OP}$ and $\mathrm{AC}$ for scenarios where we vary the background noise, the number of relays in the active relay node set and the packet size. We start with the behavior of the $\mathrm{AC}$ as the background noise is varied in Figure 2. The figure shows the comparison of multiple relay gain (MRG) on $\mathrm{AC}$ among the scenarios considering and ignoring the HPT. The MRG is the ratio between the maximal AC with multiple relays (at least has 2 relay in ANS) and the maximal AC with best single relay in ANS. The figure already illustrates the performance degradation that stems from the hardware processing delay. The system without hardware processing delay features over the entire range of the noise a gain higher than 1 . In contrast, if we introduce a hardware processing delay this leads to a significant performance degradation especially for a low background noise (always in comparison with the corresponding performance of the system with a single relay where the processing delay does not impact the system performance).

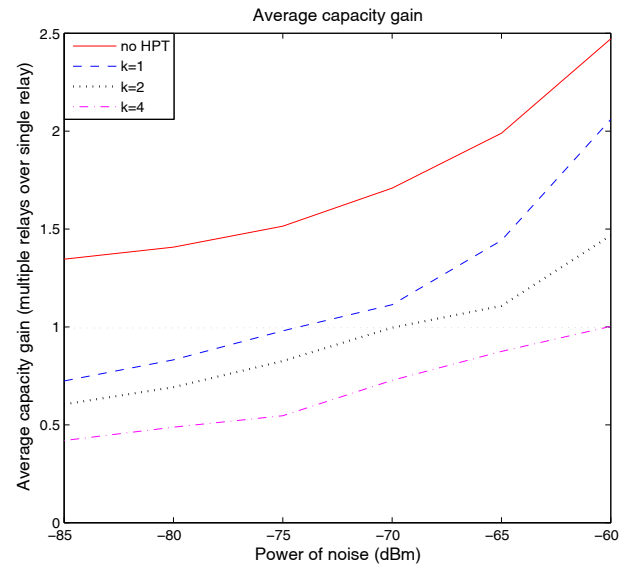

Fig. 2. AC gain of multiple relay over single relay transmission while varying the background noise among scenarios with and without considering the varying HPT $(\mathrm{k})$.

In Figure 3 we compare the OP for a packet of fixed size 2000 Bit. We vary in the figure the size of the ANS and consider the corresponding performance for four different settings of the background noise. The figure reveals the performance degradation that results from hardware processing delays. While for a low and medium background noise, the hardware processing delay of $2 \mu \mathrm{s}(k=1)$ does not affect the system performance, a higher hardware processing delay leads to a quite unreliable system performance. Furthermore, if the hardware processing delay is ignored, the OP is monotonically decreasing function of ANS size. However, the performance of the system with hardware processing delay might be either decreasing or increasing as the ANS size increases, depending on the absolute HPT but also on the background noise.

In Figure 4 we consider the $\mathrm{OP}$ as the packet size varies with the background noise. Per figure, the size of the ANS is
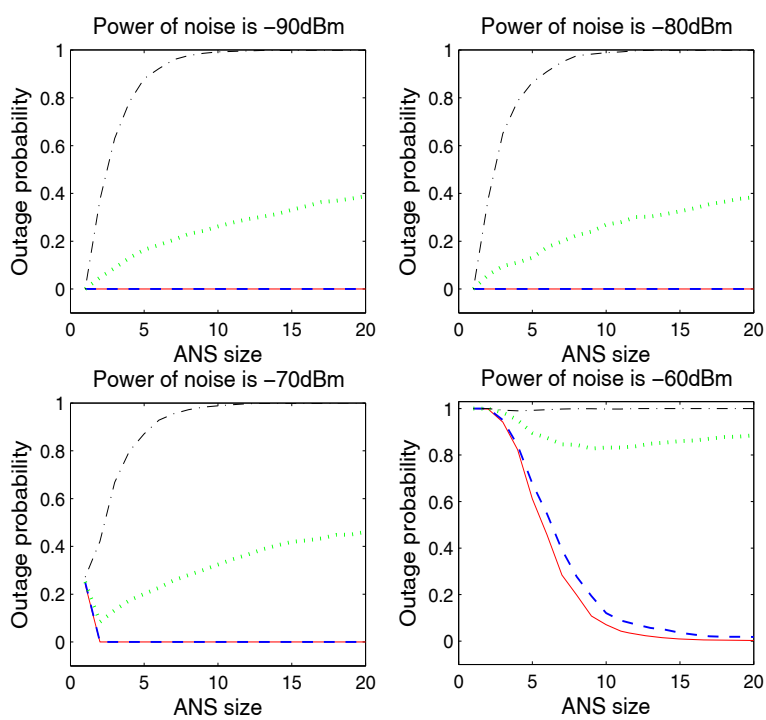

Fig. 3. OP comparison among scenarios with and without considering the synchronization mismatch with fixed packet size while considering four different settings of the background noise.
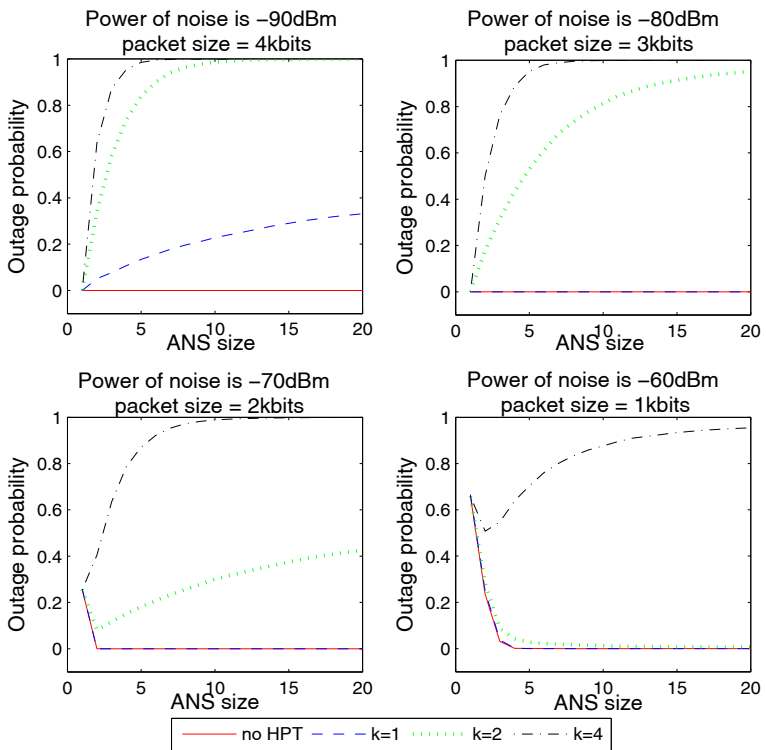

Fig. 4. OP comparison between scenarios with and without considering the synchronization mismatch while varying the background noise. For each background noise setting we consider different packet size.

varied in addition. These plots demonstrate furthermore that the hardware processing delay leads to a significant impact on the OP. For some cases, the OP first decreases (as the ANS size increases) and increases afterwards (for example, for the packet size of 2000 Bit and a background noise of $-70 \mathrm{dBm}$ ).

Overall, these presented results demonstrate the high impact of synchronization errors on the outage performance of the system. Even for a relatively small synchronization mismatch (of only $25 \%$ of the CP!) the system is already experiencing a significant performance degradation. Hence, an important question is how to cope with the timing mismatch. A simple solution is to basically just choose one relay station for forwarding. While this certainly leads to avoiding the 
synchronization problem, it also degrades the spatial diversity and SINR increase at the destination during the forwarding phase. Hence, a more promising approach is to extend the $\mathrm{CP}$ to cover all delayed path components, not only a certain fraction. Finally, as third method we investigate splitting the time for broadcasting and forwarding in an optimal manner (without adjusting the $\mathrm{CP}$ ). The next subsection contains the numerical investigation of all these three countermeasures.

\section{Studying Countermeasures}

As mentioned, we consider in the following mainly two methods to reduce the impact from hardware processing delays: Use a single relay for transmission while on the other hand considering to extend the $\mathrm{CP}$ of forwarding phase for multiple relay transmission. Both of the two methods can eliminate the ISI. However, both also bring some downsides: the single relay solution sacrifices the diversity gain of multiple relays while extending the $\mathrm{CP}$ reduces the effective packet transmission time.

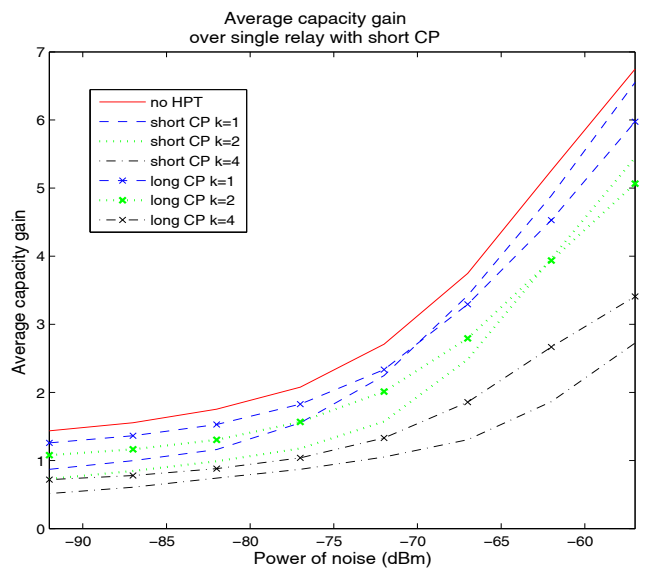

Fig. 5. AC gain: multiple relay with short/long $\mathrm{CP}$ over single relay with fixed short $\mathrm{CP}$ while varying the background noise among scenarios with and without considering the synchronization mismatch.

According to our discussion in the previous section, we first present results on the MRG of the AC of the relaying phase in Figure 5. Here, we focus now on the introduction of a longer $\mathrm{CP}$ which can cover the maximum potential delay of multi-path. As we can see from the figure, extending the $\mathrm{CP}$ typically improves the system performance if the noise power is low (i.e. in the high SNR regime) while for high background noise the performance with an extended $\mathrm{CP}$ is lower than if the $\mathrm{CP}$ is not modified. Furthermore, if the hardware processing time is large, for a low noise power (high SNR regime) it is more beneficial to only choose one relay instead of going for multiple relays.

The results are furthermore confirmed by the study of the OP in Figure 6 and 7. In these figures we compare the OP for short/long settings of the $\mathrm{CP}$ as we vary the background noise. The figures also contain the performance of the single relay system. The difference between the two figures is that they assume different packet size settings. Furthermore notice that the packet size is varied as we vary the noise power
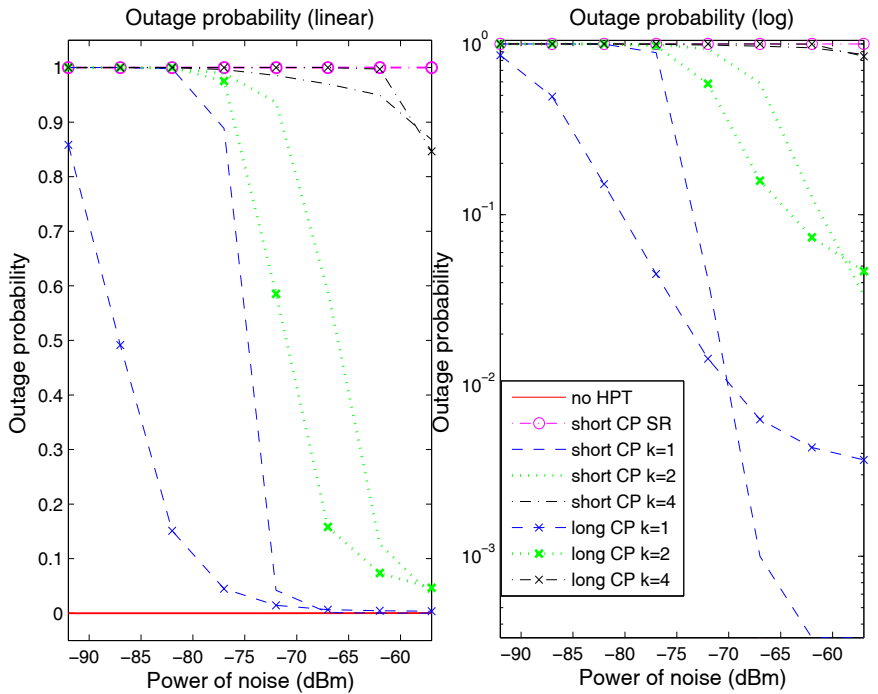

Fig. 6. OP comparison among multiple and single relay schemes while varying the background noise under scenarios with and without considering the synchronization mismatch.
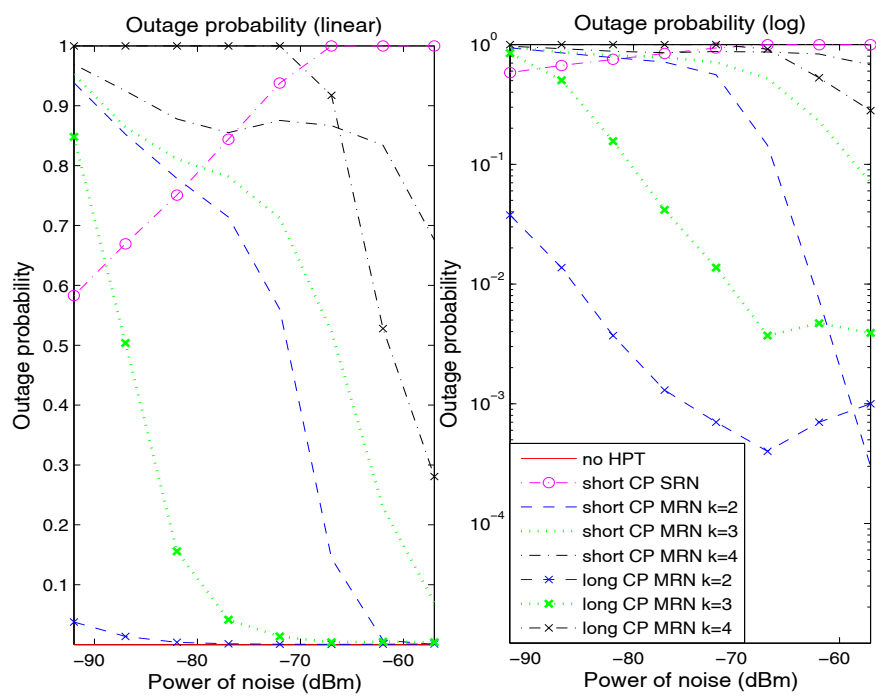

Fig. 7. OP comparison among multiple and single relay schemes only with serious HPT while varying the background noise.

(having a linear relationship with the average capacity of the relaying phase). Again we observe that each countermeasure has predominance at different range of noise. The OPs of the multiple relay system with long $\mathrm{CP}$ and short $\mathrm{CP}$ always have crossing points (i.e. there are ranges where one of them is clearly superior to the other). As the power of noise corresponds to SNR, if we treat the single relay scheme as the contrast, we can simply calculate the relative SNR of second phase based on the power of noise under the single relay scheme. For example in Figure 6 (right), when $k=1$, if the SNR of the relaying phase with single relay is bigger than 14 $\mathrm{dB}$ (equivalent to the noise power is smaller than $-70 \mathrm{dBm}$ ), it is better to go with multiple relay and extending the CP. Next, if the SNR is lower than $14 \mathrm{~dB}$, we should go with multiple relay but rather stick with the short CP. So multiple 
relay scheme is always the best choice when the system only has a slight mismatch of the processing times. However, when the mismatch of HPT is relative high (such as AC in Figure 5 and OP in Figure 7 when $k=4$ ), if the SNR is higher than 24 $\mathrm{dB}$ (equivalent to the noise power is smaller than $-80 \mathrm{dBm}$ ), it can be better to simply go with a single relay - no need to consider multiple relays in this situation. Besides, with a serious mismatch $(k=4$ in Figure 7), the performance of long $\mathrm{CP}$ and short $\mathrm{CP}$ schemes are just the opposite of slight mismatch HPT ( $k=1$ in Figure 6) scenario. Summarizing, we obtain the following statements:

If the hardware processing mismatches lead only to a marginally bigger maximum delay spread, then:

- The benefit from multi-relay association outweighs the harm from ISI even if the CP is enhanced. In other words, the multiple relay system with long CP always outperforms the single relay system.

- If the noise power is low, the ISI becomes the main cause of reducing the SINR, so the long CP scheme is suitable; while the noise is as strong as or even more stronger than ISI, the short $\mathrm{CP}$ scheme has advantages over long $\mathrm{CP}$ by saving partial overhead of $\mathrm{CP}$.

If in contrast the HPT is rather large, the ISI becomes considerable:

- The benefits from multi-relay association and the impact due to ISI on the system performance even out each other. So when the power of noise is extremely low, the singlerelay scheme outperforms the multi-relay setting.

- Since the ISI becomes considerable, long CP only has superiority over short $\mathrm{CP}$ when the noise is strong, too. Otherwise, the short $\mathrm{CP}$ is advantageous.

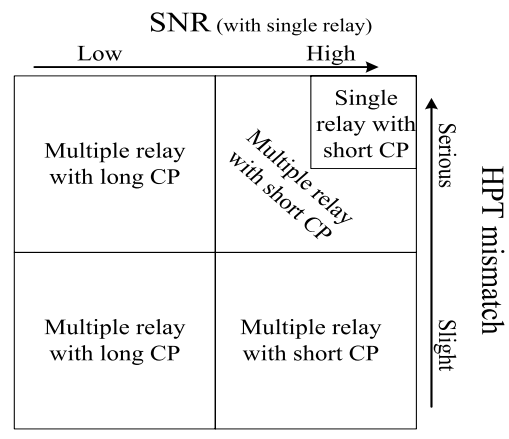

Fig. 8. The relationship among the evaluated schemes and their corresponding appropriate scope of application.

A schematic plot of the findings is also given in Figure 8. Notice in particular, that there is no single solution that improves the situation in any case but different solutions have their domains where they are the best choice.

\section{CONCLUSION}

In this paper we have focused on the performance impact of out-of-sync relays due to different HPTs. With the analysis and simulation results, this paper characterizes the performance impact of unsynchronized relays and shows a significant impact on the reliability of the system that stems from unsynchronized relays. While ignoring the HPT difference leads to the conclusion that more relays are in general beneficial, this is not the case any more if the processing time differences are taken into account. Depending on the absolute values, the HPT difference can potentially decrease the system reliability quite drastically.

Besides, this paper studies different methods to efficiently combat the synchronization issues either by introducing a longer cyclic prefix or by adjusting the number of relays to 1 , and analyzes the application scopes for these methods. With only slightly unsynchronized HPT, the long CP scheme has superiority over short CP scheme for low noise power case, while long CP schemes are advantageous if the noise power is high. This relationship is reverted when the timing mismatch becomes larger. Then the single relay scheme outperforms the other schemes when both the SNR and HPT mismatch are large.

\section{REFERENCES}

[1] Y. Kim and H. Liu, "Infrastructure Relay Transmission With Cooperative MIMO”, IEEE Trans. Vehicular Technology, Vol. 57, No. 4, pp. 21802188, Jul. 2008.

[2] Y. Fan and J. Thompson, "MIMO Configurations for Relay Channels: Theory and Practice," IEEE Trans. Wireless Commun., vol. 6, no. 5, pp. 1774-1786, May 2007.

[3] A. Adinoyi and H. Yanikomeroglu, "Cooperative Relaying in MultiAntenna Fixed Relay Networks," IEEE Trans. Wireless Commun., Vol. 6, No. 2, pp. 533-544, Feb. 2007.

[4] Y. Hu and L. Qiu, "A Novel Multiple Relay Selection Strategy for LTEAdvanced Relay Systems," in Proc. of the IEEE VTC, Budapest, Hungary, May, 2011.

[5] J. Zhang, T.M. Lok. "Performance Analysis of Multiple-Relay Decodeand-Forward Cooperation System," in Proc. of the IEEE TENCON, Melbourne, Qld, Nov. 2005.

[6] A. Bletsas, H. Shin, and M. Z. Win, "Cooperative communications with outage-optimal opportunistic relaying," IEEE Trans. Wireless Commun., vol. 6, No. 9, pp. 3450-3460, Sep. 2007.

[7] Abdelkader, A. Shahbazpanahi, S., Gershman, A. , "Joint subcarrier power loading and distributed beamforming in OFDM-based asynchronous relay networks," in Proc. of IEEE Workshop on Computational Advances in Multi-Sensor Adaptive Processing (CAMSAP), Aruba, Dutch Antilles, Dec. 2009.

[8] Elazreg, A.M., Mannai, U.N. , Chambers, J.A. ,'Distributed cooperative space-time coding with parallel interference cancellation for asynchronous wireless relay networks," in Proc. of Software, Telecommunications and Computer Networks (SoftCOM), Split-Bol, Brac, Sep. 2010.

[9] G. J. Chen, L. Ge and J. A. Chambers, "Offset Transmission Scheme with Full Interference Cancellation for An Asynchronous Cooperative Four Relay Network," in Proc. of IEEE Wireless Advanced, London, Jun. 2011

[10] R. Prasad, OFDM for wireless communications systems. Artech House Publishers, 2004.

[11] V. Erceg, K.V.S. Hari, et al, "Channel Models for Fixed Wireless Applications," IEEE Task Group Contrivutions, 802.16a-03/01, Jun. 2001.

[12] A. Hiroyuki, A. Sadayuki, S. Mamoru, "Pilot channel assisted MMSE combining in forward link for broadband OFCDM packet wireless access," IEICE Trans. on Fund. Electr., Vol. E85-A, No. 7, pp. 16351647, Jul. 2002. 